\title{
Consomme-t-on ce que l'on sème ? Relations entre diversité de la production, revenu agricole et diversité alimentaire au Burkina Faso
}

\author{
Alissia Lourme-Ruiz ${ }^{1, *}$, Sandrine Dury ${ }^{1}$ et Yves Martin-Prével ${ }^{2}$ \\ ${ }^{1}$ Cirad, UMR Moïsa, TA C-99/15, 73, rue J.F.-Breton, 34398 Montpellier cedex 5, France \\ 2 IRD, UMR Nutripass, 911, avenue Agropolis, 34394 Montpellier, France
}

\begin{abstract}
Résumé - Augmenter la production agricole peut théoriquement améliorer la diversité de l'alimentation, via l'autoconsommation ou par l'achat de produits diversifiés grâce à l'augmentation du revenu agricole. Or, dans certaines régions d'Afrique de l'Ouest où le niveau de la production est relativement élevé, on observe une faible diversité de la consommation alimentaire. L'objectif de cette étude est d'estimer, au sein de ménages agricoles, les associations entre la diversité de l'alimentation des femmes et respectivement la diversité de la production et le revenu agricole. Nous avons mené une enquête auprès de 579 exploitations représentatives de la région des Hauts-Bassins au Burkina Faso à trois périodes entre mai 2013 et janvier 2014. La diversité agricole a été évaluée à travers le nombre d'espèces cultivées, d'arbres locaux conservés sur les parcelles et d'espèces animales élevées. À partir d'un rappel qualitatif de la consommation alimentaire des 24 heures précédant l'enquête, nous avons calculé le score de diversité alimentaire des femmes en suivant les recommandations internationales récentes (nombre de groupes d'aliments consommés parmi les dix définis). Ce score est faible et ne varie pas au cours de l'année, mais les groupes d'aliments le composant changent. Les résultats d'un modèle de régression montrent que, en mai et août 2013, la diversité alimentaire n'est pas liée au nombre de cultures mais au nombre d'espèces d'arbres conservés. Les recettes monétaires agricoles des exploitations ne sont que faiblement corrélées avec une meilleure diversité alimentaire toute l'année, tandis que les recettes monétaires agricoles (mai 2013) et non agricoles des femmes le sont plus nettement. Les femmes recevant un transfert d'argent de la part du chef d'exploitation présentent par ailleurs une meilleure diversité alimentaire à toutes les saisons. Dans ce milieu rural agricole, le contrôle des ressources par les femmes semble être un meilleur garant de la qualité de leur alimentation que le niveau des productions agricoles de l'exploitation.
\end{abstract}

Mots clés : sécurité alimentaire / diversité alimentaire / plantes sauvages / production agricole / revenu / genre

\begin{abstract}
Do you eat what you sow? Linkages between farm production diversity, agricultural income and dietary diversity in Burkina Faso. An increase of agricultural production could theoretically improve dietary diversity through two main pathways: self-consumption or purchase of more diverse foods thanks to a better agricultural income. However, in some Western African regions with relatively high level of agricultural production, a low diversity of food consumption has been observed. Our objective was to assess whether the dietary diversity of women was associated with the diversity of cultivated crops and/or with sales of agricultural production at the level of the farm household in rural Burkina Faso. A representative sample of 579 farm households in the Hauts-Bassins Region was surveyed at three different periods between May 2013 and January 2014. We estimated different indicators of agriculture diversity: the number of crop species, of local tree species in the farm plots, and of animals species. From a qualitative 24-hour recall of food consumption, we computed the dietary diversity score for women according to recent international recommendations (number of food groups consumed out of 10). This score was low and did not vary across seasons, but the food groups composing it changed. Results of a multiple regression showed that, in May and August 2013, women dietary diversity was not associated with the number of crops but with the number of
\end{abstract}

\footnotetext{
* Auteur de correspondance : alr.anglais@gmail.com,

alissia.lourme_ruiz@cirad.fr
} 
local spared tree species. Sales value was weakly related with a better dietary diversity at all seasons, while the association was stronger with women agricultural (in May 2013) and non-agricultural incomes. At all seasons, women receiving cash transfers from the head of farm household had far higher dietary diversity scores than those who did not. Finally, women control over resources seems to better warrant women dietary diversity that the level of agricultural production at the farm household level.

Keywords: food security / dietary diversity / wild plants / agricultural production / income / gender

\section{Introduction et objectifs}

Dans certaines régions rurales d'Afrique de l'Ouest, un niveau relativement élevé de production agricole coexiste avec une forte prévalence du retard de croissance des jeunes enfants (céréales et coton au Mali :Tefft et al., 2000 ; Dury et Bocoum, 2012 ; produits maraîchers au Burkina : Parent et al., 2002). Le retard de croissance est la manifestation chronique de la sousnutrition infantile, provenant d'une accumulation de carences alimentaires et de pathologies infectieuses, en lien avec les faibles ressources et capacités des ménages. En outre, la faible diversité de l'alimentation est reconnue comme une des causes importantes de la malnutrition ; en milieu rural, à l'Ouest du Burkina Faso, Arsenault et al. (2014) ont montré que l'alimentation des enfants et des femmes était pauvre en plusieurs micronutriments (vitamines et minéraux).

Une production agricole abondante, régulière et diversifiée, tout comme l'élevage, pourrait théoriquement permettre l'accès à une alimentation plus diversifiée au sein des ménages agricoles via deux chemins principaux : l'autoconsommation et/ou les revenus issus de la vente de la production, permettant d'acheter des aliments plus variés (Carletto et al., 2015). Mais, pour un chemin comme pour l'autre, les liens sont complexes et les résultats des quelques études empiriques ne convergent pas forcément dans leurs conclusions.

Tandis que certains travaux observent une relation positive entre la diversité de la production et la diversité alimentaire mesurée au niveau des ménages agricoles (Bhagowalia et al., 2012 en Inde ; Jones et al., 2014 au Malawi), d'autres ne trouvent pas d'association (Torheim et al., 2004 avec la diversité alimentaire individuelle à l'Ouest du Mali). Sibhatu et al. (2015), à partir de données d'Indonésie, du Kenya, du Malawi et d'Éthiopie, suggèrent que, dans le cas d'un nombre déjà élevé de cultures produites, le lien avec la diversité alimentaire peut être nul ou négatif.

Concernant le revenu, dans une étude menée au Tchad Bégin et al. (1999) ont montré que les enfants avaient un meilleur statut nutritionnel quand une part de la production était vendue. Dillon et al. (2014) ont montré cependant qu'un revenu agricole plus élevé n'était associé que très faiblement à la diversité de la consommation alimentaire des ménages au Nigéria.

Enfin, la répartition des ressources à l'intérieur des ménages est importante, puisque l'augmentation du revenu des femmes améliorerait leur pouvoir de décision au sein du ménage et augmenterait les dépenses pour l'alimentation et le soin de la famille (Hoddinott et Haddad, 1995 en Côte d'Ivoire). Par ailleurs, Kelly et al. (2004) observent au Mali que les enfants des zones cotonnières souffrent plus de retard de croissance que ceux d'autres zones, évoquant parmi les causes possibles la faible autonomie financière des femmes et le fait que le revenu soit concentré dans les mains des hommes les plus vieux des exploitations.
Malgré des indicateurs de malnutrition et d'insécurité alimentaire inquiétants en Afrique de l'Ouest, à notre connaissance, aucune étude empirique ne s'est intéressée aux relations entre la diversité de la production agricole et la diversité de la consommation alimentaire individuelle. Des recherches plus approfondies sont nécessaires pour promouvoir des interventions agricoles bénéfiques pour la santé et la nutrition, dans la mesure où la plupart des politiques et projets de sécurité alimentaire recommandent d'augmenter les quantités produites, avec peu d'attention portée à l'amélioration de la qualité nutritionnelle (diversité et équilibre de l'ensemble des nutriments) des systèmes de productions agricoles (Miller et Welch, 2013). Le lien spécifique entre production agricole et diversité de la consommation alimentaire des individus au sein des ménages agricoles en Afrique de l'Ouest constitue à la fois un enjeu scientifique et un enjeu de développement.

L'objectif de cette étude est d'identifier les relations entre la diversité de la production agricole, les recettes monétaires issues de la vente des produits agricoles et la diversité de l'alimentation des mères de jeunes enfants.

\section{La région des Hauts-Bassins : production et malnutrition élevées}

La région des Hauts-Bassins, située à l'Ouest du Burkina Faso (Fig. 1), est reconnue pour ses productions céréalières et cotonnières abondantes par rapport à d'autres régions. La production annuelle moyenne était de $412 \mathrm{~kg}$ de céréales (sorgho, mil, maïs, fonio) par habitant et par an entre 2007 et 2011, contre $261 \mathrm{~kg}$ dans le pays (Fig. 2). Par ailleurs, cela correspond à plus du double du seuil d'autosuffisance alimentaire fixé à $190 \mathrm{~kg}$ par habitant par le Comité permanent inter-États de lutte contre la sécheresse dans le Sahel (Cilss, 2004). Dans le même temps, $39 \%$ des enfants de moins de 5 ans de cette région souffraient de retard de croissance en 2011 (enquête nutritionnelle nationale du Burkina Faso).

\section{Méthodologie}

La méthode que nous avons suivie consiste à observer, par enquête, à la fois la diversité de la production (incluant, au-delà des différentes cultures, les espèces animales élevées et les arbres locaux intéressants pour l'alimentation, conservés et entretenus sur les parcelles cultivées), les revenus des exploitations agricoles, les revenus individuels et la diversité de l'alimentation des mères de jeunes enfants vivant sur ces exploitations agricoles. Nous avons évalué statistiquement les relations entre ces différentes variables, en tenant compte des autres facteurs d'interactions.

Après une phase exploratoire qualitative menée de février à mai 2013 au cours de laquelle un film documentaire a été réalisé 


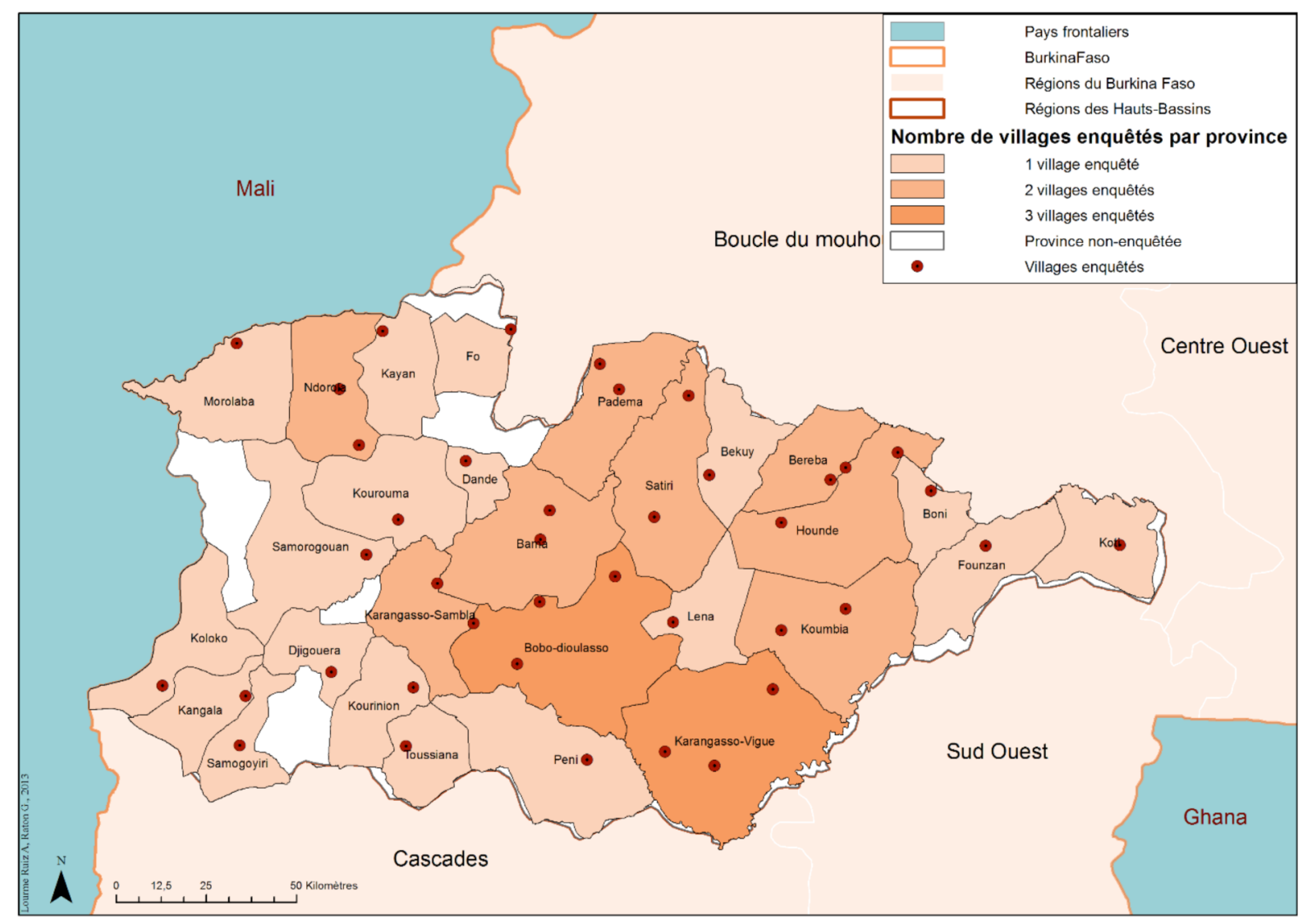

Fig. 1. Situation des villages étudiés dans les communes des Hauts-Bassins.

Fig. 1. Location of surveyed villages in the municipalities of the Hauts-Bassins.

(Lourme-Ruiz et Maugérard, 2014), une enquête quantitative par questionnaire fermé a été menée auprès d'un échantillon représentatif de 600 exploitations agricoles de la région administrative des Hauts-Bassins.

À partir de la base nationale de recensement de la population de 2006, 40 villages (Fig. 1) ont été tirés au sort de façon proportionnelle au poids de leur population. Puis, nous avons recensé les exploitations de chaque village afin de tirer aléatoirement un nombre fixe de 15 exploitations agricoles par village afin d'obtenir un échantillon auto-pondéré. Au sein de chaque exploitation agricole ont ensuite été inclus pour l'enquête le chef d'exploitation, l'enfant le plus jeune de l'exploitation parmi ceux âgés de 6 à 24 mois (au moment de la première enquête) et sa mère.

Nous avons effectué trois passages afin de tenir compte des variations saisonnières de la production et de la consommation alimentaire : en saison sèche, plusieurs mois après les récoltes (mai 2013) ; en période de soudure, après les semis pendant la saison des pluies lorsque les greniers de céréales sont souvent vides (août 2013) ; et en période post-récolte (janvier 2014).

Nous avons conservé pour l'analyse 579 exploitations lors du premier passage car 21 observations montraient des incohérences. Puis, nous avons retrouvé 402 exploitations au deuxième passage et 421 au troisième passage. Nous avons cessé le suivi auprès de 15 exploitations suite au décès de l'enfant. Nous avons comparé les caractéristiques mesurées au premier passage entre les individus enquêtés à tous les passages et ceux non retrouvés aux passages 2 et 3 . Il n'y avait pas de différence significative entre ces groupes, sauf pour la variable des transferts monétaires des chefs d'exploitation aux femmes (parmi les femmes absentes en août 2013, $26 \%$ n'avaient pas reçu de transferts monétaires en mai 2013 contre $19 \%$ parmi les femmes retrouvées ; $p=0,04$ ).

\subsection{Mesure de la diversité alimentaire}

À partir d'un rappel qualitatif de la consommation des dernières 24 heures, nous avons calculé le «score de diversité alimentaire des femmes », qui est un indicateur de la couverture des besoins en micronutriments (Martin-Prével et al., 2015). Ce score correspond au nombre de groupes d'aliments consommés par les femmes parmi les dix groupes suivants :

- les aliments amylacés (surtout le maïs et le sorgho dans cette région) ;

- les pois et les haricots (le pois voandzou, le niébé...) ;

- les noix et les graines (l'arachide, le sésame...);

- les produits laitiers ;

- les chairs animales (viandes, abats et poissons) ;

- les œufs ;

- les légumes feuilles riches en vitamine A (feuilles de baobab, oseille de Guinée...) ;

- les autres fruits et légumes riches en vitamine A (mangue...) ;

- les autres légumes (tomate, oignon...) ;

- les autres fruits (banane...). 


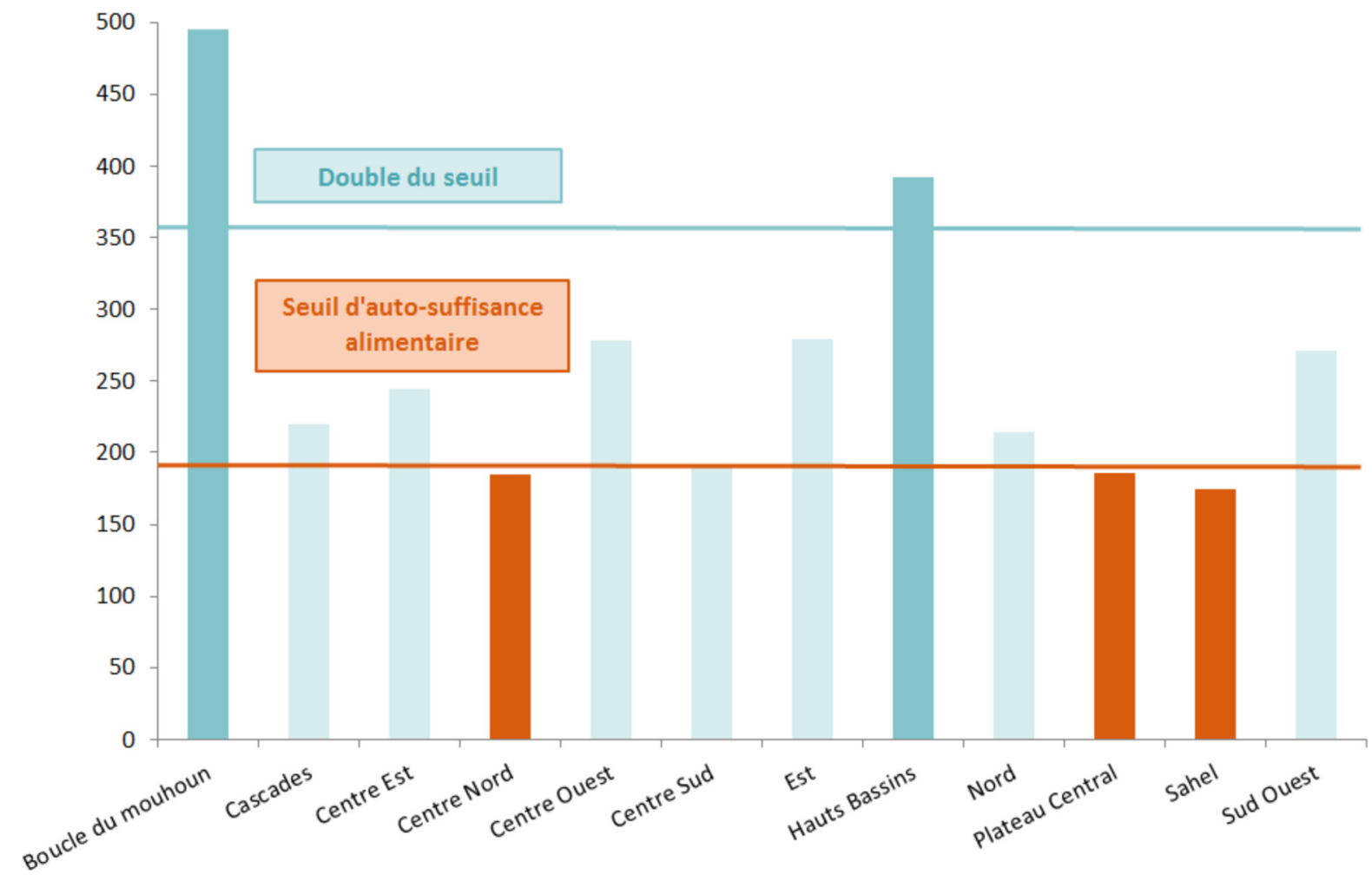

Fig. 2. Production de céréales par habitant et par an (moyenne 2009-2011) dans les différentes régions administratives du Burkina Faso.

Fig. 2. Production of cereals per capita per year (mean 2009-2011) in the different administrative regions of Burkina Faso.

Source : graphe et calculs des auteurs à partir des données de production de céréales de 2009 à 2011 de la Direction générale de la promotion de l'économie rurale (DGPER). Seuil d'autosuffisance alimentaire $=190 \mathrm{~kg} / \mathrm{an}$ par personne (Cilss, 2004)

Le seuil de cinq groupes est celui à partir duquel on a, dans la population, un pourcentage nettement plus important de femmes couvrant leurs besoins nutritionnels de façon satisfaisante (Martin-Prével et al., 2015).

\subsection{Mesure des données agricoles}

Pour l'exploitation familiale puis pour les champs personnels de la mère, nous avons listé les types de culture et les volumes récoltés pour la campagne 2012-2013 (en mai 2013) et pour la campagne 2013-2014 (en janvier 2014). En janvier 2014, certaines productions (notamment fruitières arboricoles) n'avaient pas été récoltées et certaines exploitations n'avaient pas encore vendu leur coton ; aussi, les données de récolte et de vente sont incomplètes pour 2013-2014 et non utilisées dans les analyses.

Les quantités étaient exprimées en unités locales de mesure (ULM : sac, boîte de conserve...) lors des entretiens. Nous les avons converties ensuite en kilogrammes en adaptant les tables de conversion utilisées par le ministère de l'Agriculture pour les enquêtes permanentes agricoles.

\subsubsection{Mesure du revenu agricole}

Nous avons mesuré le revenu agricole au niveau des exploitations et des femmes, quand elles avaient une parcelle en responsabilité ; il est estimé par le montant des ventes des denrées (y compris animales) produites depuis le début de la campagne agricole (environ dix mois). Cet indicateur de revenu ne valorise pas les quantités produites et non vendues au moment de l'enquête (stockées, autoconsommées, données ou perdues). Il reflète la disponibilité monétaire issue de la production mais pas la valeur de la production totale.

\subsubsection{Mesure de la diversité agricole}

Afin de cerner plusieurs dimensions de la diversité des sources de produits alimentaires cultivés, élevés ou cueillis sur l'exploitation agricole, nous avons utilisé trois indicateurs :

- le nombre de cultures (annuelles ou pérennes) alimentaires produites : mil, maïs, riz, fonio, sorgho, arachide, sésame, soja, niébé, voandzou, igname, patate, taro, manioc, agrumes, anacarde, banane, mangue, papaye, oignon bulbe, oignon feuille, chou, tomate, aubergine, piment, haricot vert, concombre, poivron, gombo, courgette, pastèque/ melon, gingembre, oseille de guinée ;

- le nombre d'espèces d'arbres locaux intéressants pour l'alimentation conservés volontairement sur les parcelles ; parmi les espèces identifiées, on note le baobab (Adansonia digitata), le karité (Vitellaria paradoxa), le néré (Parkia biglobosa), le «mimosa » (Acacia macrostachya), le kapokier rouge (Bombax costatum), le tamarinier (Tamarindus indica), le raisin sauvage (Lannea microcarpa), le rônier (Borassus) et le " prunier » (Vitex doniana) ;

- le nombre d'espèces animales élevées, hors animaux de traction. Ces espèces ont été regroupées en cinq grands types : bovins, ovins et caprins, équins et asins, volailles, et porcins. 


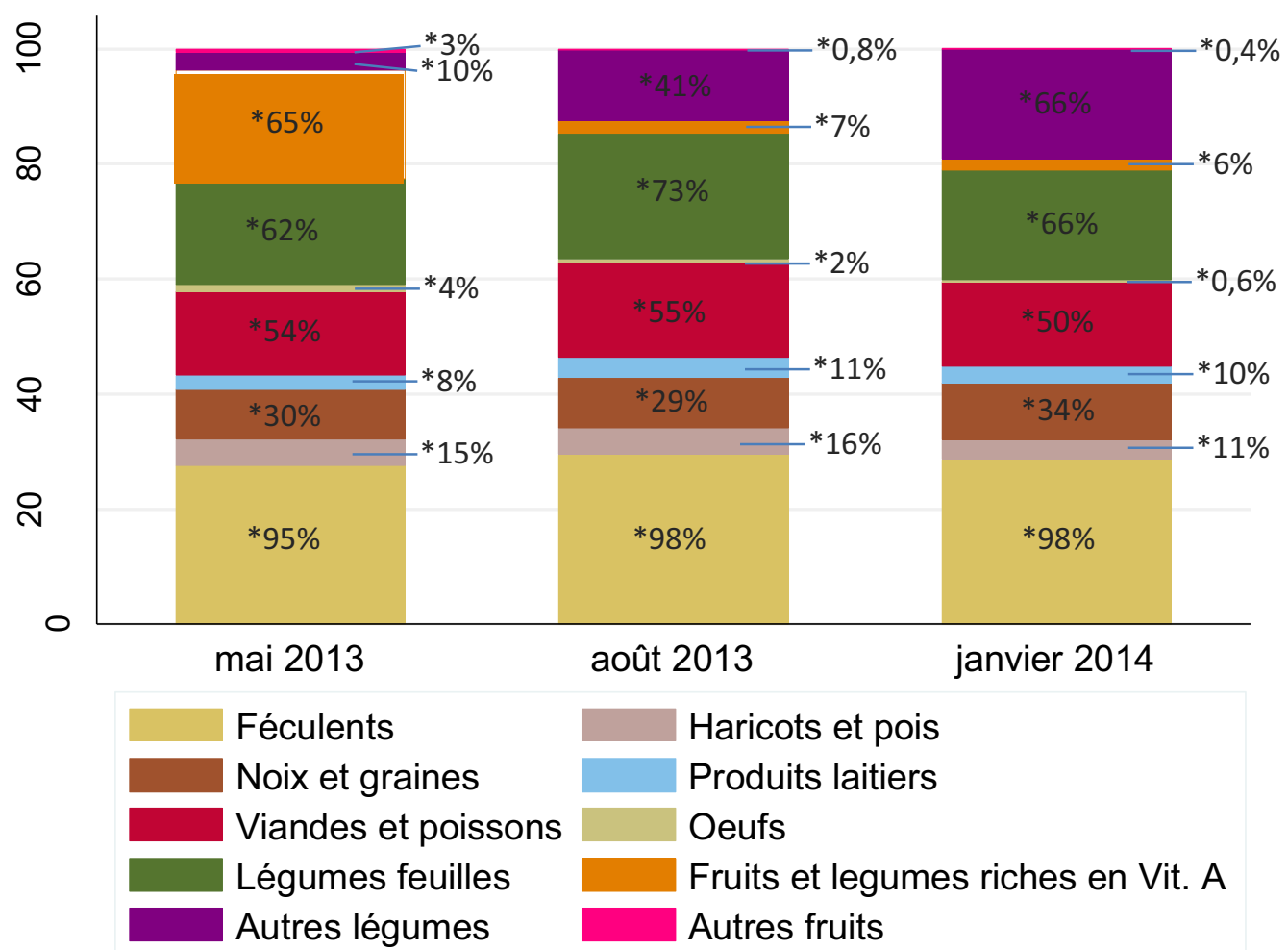

Fig. 3. Groupes d'aliments consommés selon la saison. * Le pourcentage de femmes ayant consommé le groupe alimentaire. Fig. 3. Share of food groups across seasons.

\subsection{Mesure des covariables}

Nous avons également pris en compte les revenus non agricoles des chefs d'exploitation et des femmes pour le mois précédant l'enquête. Ces revenus sont exprimés par tranches (0, inférieur ou supérieur à 10000 FCFA, soit environ 15 euros), car ils sont difficiles à évaluer de façon plus précise. De plus, nous avons inclus les transferts monétaires du chef d'exploitation vers les femmes sous forme de variable dichotomique.

\subsection{Méthode analytique}

Nous avons estimé un modèle de régression linéaire multiple avec Stata 12 (StataCorp, 2011) afin d'identifier les déterminants de la diversité alimentaire des femmes. Les variables indépendantes d'intérêt sont la diversité de la production et la valeur des ventes de produits agricoles. Nous avons intégré des covariables explicatives, économiques, socioculturelles, environnementales (taille des ménages, âge de la femme, son alphabétisation et distance au marché) correspondant aux déterminants de l'alimentation des femmes décrits dans la littérature. Les coefficients de ces régressions sont présentés dans le Tableau 3.

\section{Résultats}

\subsection{La diversité alimentaire des femmes est faible et stable mais les groupes d'aliments consommés varient selon la saison}

4.1.1 La diversité alimentaire des femmes repose sur les « sauces » et les « encas »

Les plats familiaux sont constitués de tô (une pâte épaisse préparée avec de la farine de céréale et de l'eau) et, comme c'est le cas généralement en Afrique de l'Ouest (Bricas et Akindes, 2012), d'une sauce. Cette sauce joue un rôle fondamental dans la variété des nutriments fournis par l'alimentation. En milieu rural, la sauce n'est généralement pas très riche, telle la simple « sauce feuille » composée de légumes feuilles (de baobab par exemple), d'huile et de sel, mais elle peut aussi parfois être agrémentée de pâte d'arachide, de légumes, de poisson ou de morceaux de viande. En plus des plats familiaux, les femmes consomment parfois et selon les saisons, en dehors des repas, des " encas », issus de la cueillette (comme la mangue), ou bien « grignotés » au moment de la cuisine. Par exemple, elles peuvent consommer de la poudre de néré pendant qu'elles fabriquent le soumbala - une préparation culinaire - ou des arachides en préparant la sauce.

\subsubsection{La diversité alimentaire des femmes est faible et stable d'une saison à l'autre}

Quelle que soit la période (mai 2013, août 2014 ou janvier 2014), la diversité alimentaire des femmes est très faible (Tab. 1). La majorité des mères $(80 \%)$ n'atteint pas le seuil recommandé de cinq groupes d'aliments consommés. Les femmes consomment en moyenne $3,45 \quad( \pm 1,47)$ groupes d'aliments en saison sèche, $3,35( \pm 1,3)$ groupes en période de soudure et 3,44 $( \pm 1,29)$ groupes en période post-récolte. Le test de comparaison de moyennes ne montre pas de différence significative entre les saisons.

\subsubsection{La composition des groupes d'aliments consommés varie selon la saison}

Les groupes d'aliments consommés varient entre les trois passages (Fig. 3). Ainsi, en mai 2013, en période des mangues, 
Tableau 1. Caractéristiques de l'échantillon.

Table 1. Sample characteristics.

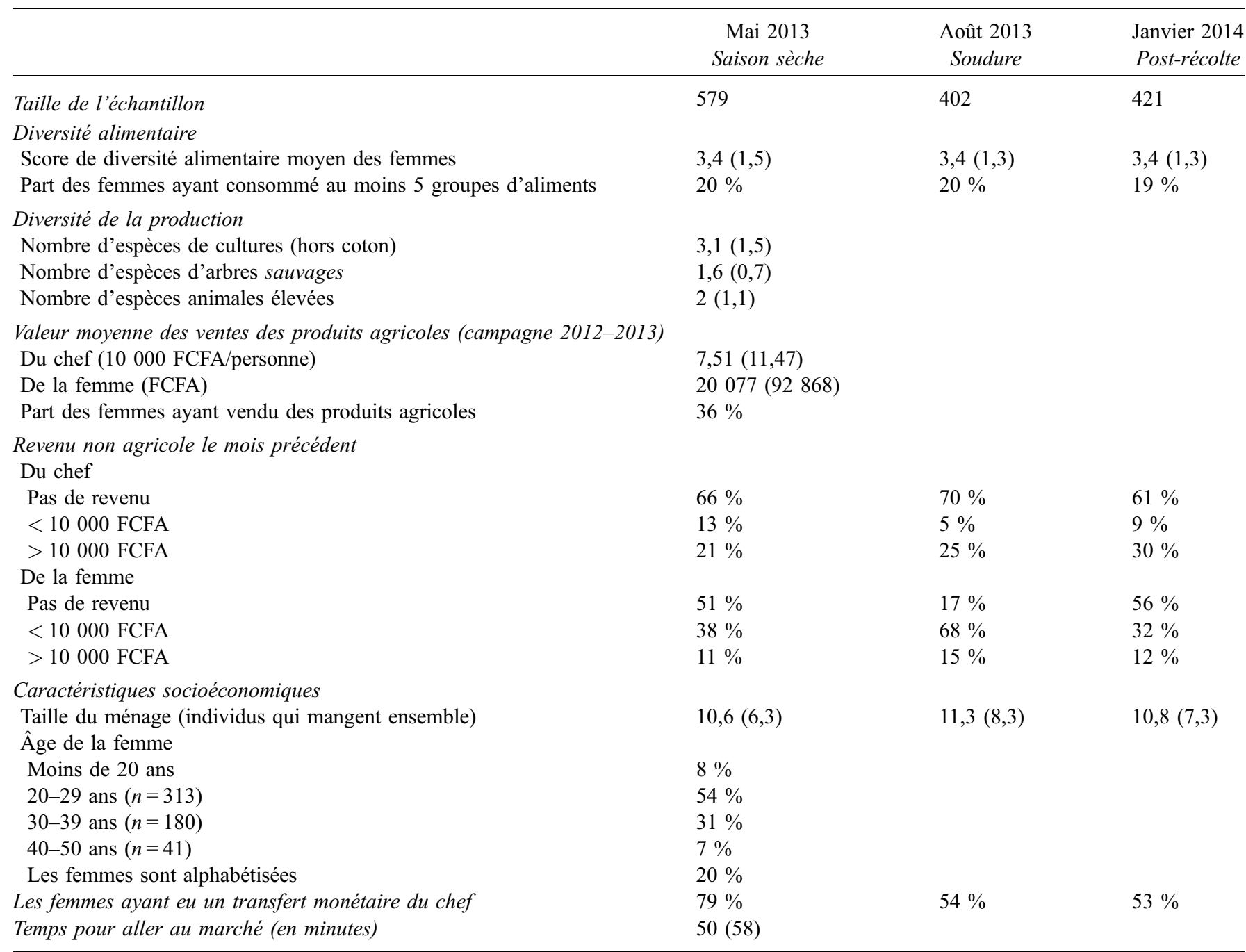

Pour les variables continuent, nous présentons la moyenne (l'écart-type).

Pour les variables catégorielles, nous présentons le pourcentage de la catégorie.

$65 \%$ des femmes ont consommé des fruits riches en vitamine $A$, contre seulement $7 \%$ en août 2013 et $6 \%$ en janvier 2015. Les légumes feuilles verts foncés (riches en vitamine A) sont un peu plus consommés en août 2013 qu'aux deux autres périodes (73\% des femmes contre $62 \%$ en mai 2013 et $66 \%$ en janvier 2014). Les légumes issus du maraîchage sont très peu consommés en mai 2013 : seules $10 \%$ des femmes sont concernées alors qu'elles sont $41 \%$ en août 2013 et $66 \%$ en janvier.

Les féculents (céréales et tubercules) sont logiquement consommés par quasiment la totalité des femmes quelle que soit la période. Les consommations de légumineuses (pois et haricots), d'oléagineux (noix et graines) et de chairs animales (viandes et poissons) restent stables. La consommation des autres produits d'origine animale (lait, œufs...) et de fruits (hors mangues) est marginale.

Lorsque les femmes consomment plus de groupes d'aliments, ce sont les produits laitiers, les noix et les graines (notamment dans les sauces à base d'arachide) ou encore la viande dont la fréquence augmente.

\subsection{La production agricole est « relativement 》 élevée, source de calories, mais peu diversifiée}

4.2.1 Des systèmes de cultures annuelles peu diversifiés dominés par le coton et le maïs

Les exploitations cultivent en moyenne 3,1 $( \pm 1,5)$ types de cultures différentes, hors coton (Tab. 1). Toutes les exploitations $(99 \%)$ cultivent du maïs, $47 \%$ cultivent du maïs et une autre céréale, $27 \%$ du maïs et deux autres céréales.

Le maïs et le coton sont produits à la fois par de nombreux agriculteurs et en grandes quantités. Les deux tiers des exploitations de l'échantillon produisent du coton en 2012-2013 (Tab. 1). La production moyenne de maïs atteint 
Tableau 2. Production agricole par exploitation (campagne 2012-2013).

Table 2. Agricultural production per exploitation (season 2012-2013).

\begin{tabular}{llcc}
\hline Culture & $\begin{array}{l}\text { Nombre d'exploitations } \\
\left(\mathrm{en} \%^{\mathrm{a}}\right)\end{array}$ & $\begin{array}{l}\text { Production disponible } \\
\text { (moyenne sur l'échantillon } \\
\text { en kg/personne) }\end{array}$ & $\begin{array}{l}\text { Part de la production } \\
\text { vendue }\end{array}$ \\
\hline Céréales & & 486 \\
Dont maïs & 99 & 397 & 22 \\
Coton & 93 & 267 & 25 \\
Oléagineux et légumineuses & 67 & 33 & 37 \\
Racines et tubercules & 51 & 38 & 93 \\
Maraîchage & 7 & 20 & 87 \\
Arboriculture & 8 & 103 & 95 \\
\hline
\end{tabular}

${ }^{\text {a }}$ Sur les 579 exploitations enquêtées au premier passage (mai 2013).

${ }^{\mathrm{b}}$ Calcul effectué sur la base de l'estimation des quantités produites et vendues par le chef d'exploitation.

$487 \mathrm{~kg}$ par personne en 2013, soit un équivalent de 3970 calories par personne et par jour. Ainsi, s'il était entièrement consommé, le maïs suffirait largement - en moyenne - à couvrir à lui seul les besoins énergétiques des individus.

La moitié des exploitations produisent des oléagineux et/ou des légumineuses (arachide, niébé, voandzou essentiellement), mais les volumes sont faibles (Tab. 2). En moyenne $33 \mathrm{~kg}$ d'oléagineux et de légumineuses, $38 \mathrm{~kg}$ de racines et de tubercules et $20 \mathrm{~kg}$ de produits maraîchers sont disponibles par habitant et par an dans la région. Seules $11 \%$ des exploitations pratiquent l'arboriculture. Elles sont concentrées dans le Sud-Ouest de la région, appelé le verger du Burkina.

Par ailleurs, la plus grande partie des racines et tubercules, des produits maraîchers et des fruits (respectivement 93, 87 et $95 \%$ des quantités récoltées) sont vendus (Tab. 2), donc ceuxci ne sont pas autoconsommés par les ménages.

\subsection{2 ... associés à des arbres et des animaux}

Le nombre d'arbres locaux conservés sur les parcelles est en moyenne de 1,6 $( \pm 0,7)$ par exploitation agricole, ce qui est, relativement au nombre de cultures, important.

Les exploitations possèdent en moyenne deux types d'animaux, des volailles, des bovins ou des ovins ; $92 \%$ des exploitations possèdent des animaux d'élevage et on compte en moyenne 27 animaux par exploitation, dont deux animaux de traction.

\subsubsection{Des revenus monétaires agricoles basés sur le coton, complétés par des revenus non agricoles}

Pendant la campagne 2012-2013, la vente des produits agricoles des exploitations a représenté en moyenne 75172 $( \pm 114783)$ FCFA par personne. Ce montant est très faible car il correspond à environ 280 FCFA par jour par personne, c'est-àdire à 0,5 euros. Au sein de l'échantillon, le coton représente $54 \%$ de cette valeur, le maïs $17 \%$, les mangues et les anacardes, $11 \%$.

Parmi les chefs d'exploitation, $34 \%$ ont un revenu non agricole en saison sèche (mai 2013), $35 \%$ en période de soudure (août 2013) pendant les travaux champêtres et $39 \%$ en période post-récolte (janvier 2014) ; une majeure partie ont gagné plus de 10000 FCFA au cours du mois précédent l'enquête. Les femmes sont plus nombreuses que les chefs d'exploitation à avoir gagné un peu d'argent dans une activité différente de la vente de produits agricoles ; elles sont $49 \%$ en mai 2013, $83 \%$ en août 2013 et $44 \%$ en janvier 2014, en période de post-récolte. Mais la plupart d'entre elles ont gagné moins de 10000 FCFA.

\subsection{Les déterminants de la diversité alimentaire}

4.3.1 Diversité de la production agricole et diversité de la consommation alimentaire

Le nombre de cultures alimentaires produites n'est pas significativement lié à la diversité des groupes d'aliments consommés, et ce quelle que soit la saison considérée (Tab. 3).

En revanche, le nombre d'espèces d'arbres locaux d'intérêt alimentaire est significativement lié au nombre de groupes d'aliments consommés par les femmes en mai et en août 2013. Lorsqu'une espèce d'arbre supplémentaire est présente dans les parcelles, alors les femmes consomment respectivement $0,197(p<0,05)$ et $0,137(p<0,1)$ groupes d'aliments de plus.

Enfin, le nombre d'espèces animales élevées est négativement corrélé avec la diversité alimentaire des femmes pendant la saison pluvieuse (en août 2013).

\subsubsection{Le revenu monétaire de l'exploitation agricole et la diversité alimentaire sont liés}

Les ventes de produits agricoles de la campagne 2012-2013 sont significativement corrélées avec la diversité alimentaire des femmes en toutes saisons mais les valeurs des coefficients sont faibles. Lorsque les ventes augmentent de 10000 FCFA par personne, les femmes consomment respectivement $0,009(p<0,1), 0,018$ et $0,022(p<0,001)$ groupes d'aliments supplémentaires.

Le revenu agricole des femmes est associé à leur diversité alimentaire en mai 2013, et plus fortement que celui des chefs d'exploitation. Quand une femme vend des produits agricoles, elle consomme $0,214(p<0,1)$ groupes d'aliments en plus. 
Tableau 3. Régression multiple sur la diversité alimentaire des femmes (MDD-W).

Table 3. Multiple regression results of determinants of women dietary diversity.

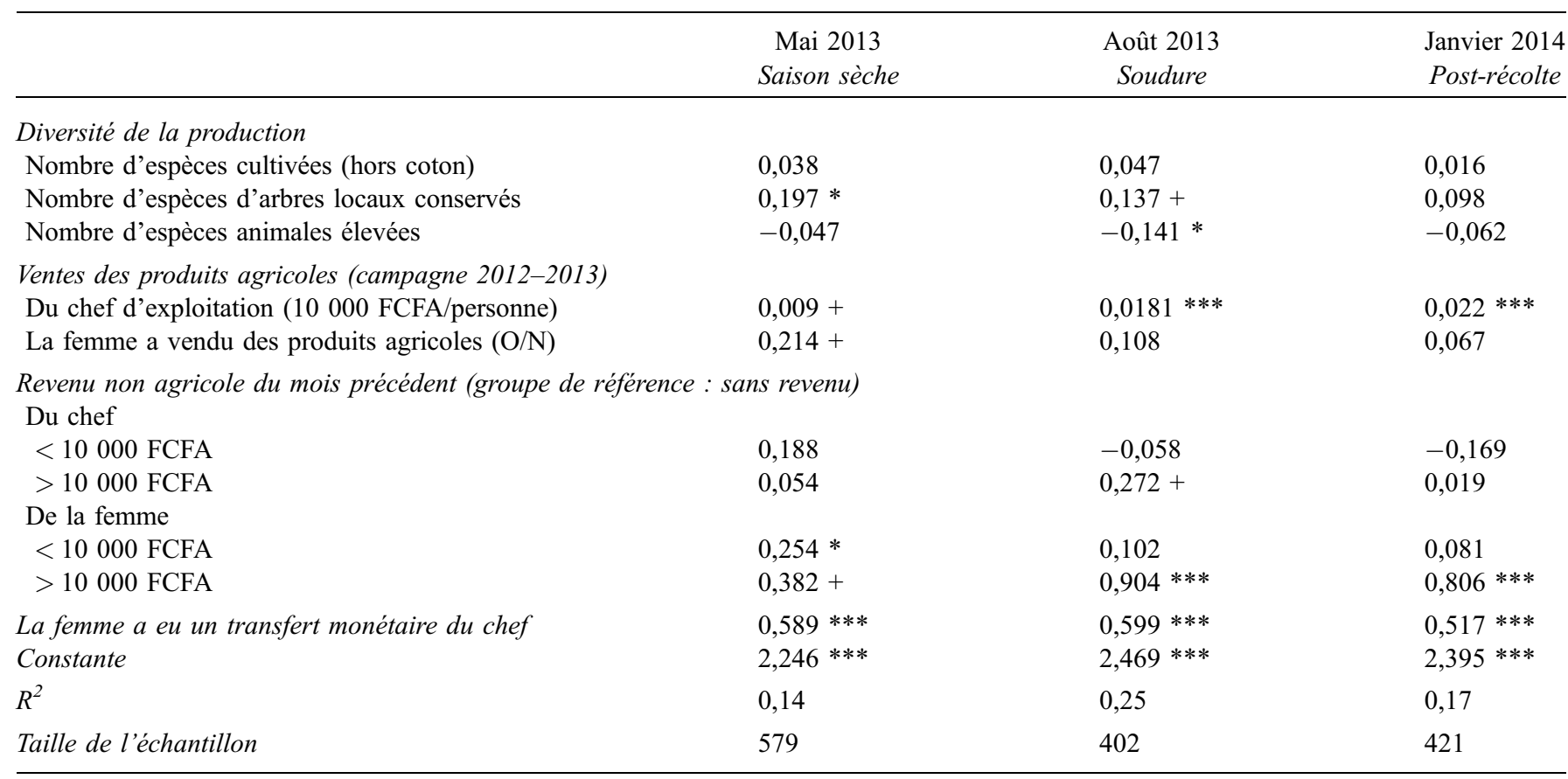

Les valeurs correspondent aux coefficients des variables d'intérêt de la régression multiple.

Les variables d'ajustement utilisées sont : la taille du ménage, l'âge de la femme, la femme est alphabétisée et la distance au marché.

$+p<0,10 ; * p<0,05 ;{ }^{* *} p<0,01 ; * * * p<0,001$.

\subsubsection{Les variables économiques et sociales ont un effet positif, surtout celles liées aux femmes}

Le revenu non agricole des femmes a un effet positif, stable et fort sur leur diversité alimentaire à toutes les saisons quand ce revenu est supérieur à 10000 FCFA le mois précédant l'enquête ; mais aussi, uniquement en mai 2013, même si ce revenu est inférieur à $10000 \mathrm{FCFA}$. En outre, les femmes ayant touché un revenu supérieur à 10000 FCFA consomment 0,382 $(p<0,1)$ groupes alimentaires de plus que les femmes n'ayant eu aucun revenu en saison sèche, $0,904(p<0,001)$ en période de soudure et $0,806(p<0,001)$ en période post-récolte.

Les revenus non agricoles des chefs d'exploitation sont liés avec la diversité alimentaire des femmes en août 2013, pour des revenus supérieurs à 10000 FCFA.

Les transferts monétaires des chefs d'exploitation vers les femmes pour les dépenses familiales (pour la plupart alimentaires) sont liés de façon stable et significative à la diversité de la consommation des femmes (plus de 0,5 $[p<0,001]$ groupes d'aliments consommés en plus).

\section{Discussion}

Dans notre étude, la diversité de la consommation alimentaire des femmes n'est pas liée au nombre de cultures alimentaires de l'exploitation (Tab. 3). Comme nous avions fait l'hypothèse que la diversité alimentaire était très liée à celle de la production agricole, nous avons pensé que notre résultat était peut-être dû au fait que la méthode de construction de l'indicateur de la diversité des cultures était trop différente de celle de la diversité de l'alimentation. Aussi avons-nous testé différents indicateurs de la diversité des cultures, plus ou moins complexes. En particulier, nous avons calculé un indicateur qui tenait compte des quantités relatives des cultures (indice de Simpson). Mais nous n'avons pas mis en évidence de relation entre l'indicateur de diversité des cultures et celui de l'alimentation.

Ce manque de relation peut s'expliquer par deux raisons :

- les systèmes de production sont peu diversifiés si l'on considère les groupes d'aliments. Plusieurs cultures différentes peuvent en effet appartenir au même groupe : par exemple, la quasi-totalité des exploitations produisent des céréales ; $47 \%$ cultivent deux céréales et $27 \%$ plus de trois céréales différentes ;

- une partie importante de la production non céréalière est vendue (Tab. 2) et donc l'autoconsommation n'est pas le principal mode d'approvisionnement pour ces types d'aliments.

Dans l'enquête alimentaire, nous avons demandé quel était le mode principal d'acquisition de chacun des groupes d'aliments consommés. Pour 51,35 et $51 \%$ d'entre eux, respectivement en mai 2013, août 2013 et janvier 2014, l'achat est le mode essentiel d'acquisition. De plus, la production est le principal mode d'acquisition pour seulement $38 \%$ des groupes d'aliments consommés en mai 2013 , les $11 \%$ restant provenant des dons perçus et de la cueillette. Ces résultats sont surprenants par rapport à l'a priori selon lequel l'autoconsommation est très importante chez les ménages agricoles de cette région. Cela est vrai si l'on considère le groupe des céréales, et donc la couverture des besoins en calories, puisque $84 \%$ de leur consommation est issue de la production, et $11 \%$ des achats, en mai 2013. En revanche, les 
aliments des autres groupes, souvent plus variés en nutriments, proviennent del'exploitation agricole dans moins de $30 \%$ des cas.

Le nombre d'espèces animales élevées est négativement lié à la diversité de l'alimentation des femmes en mai 2013, au moment où les travaux agricoles sont les plus intenses. Ce lien négatif est difficile à expliquer simplement, et nous avons cherché si des variables omises dans notre régression pourraient être liées à la fois à la diversité alimentaire et au nombre d'espèces animales élevées. Par exemple, les animaux pourraient devenir une réelle contrainte en termes de charge de travail pour les femmes pendant cette période et entraveraient la préparation de plats diversifiés. Par ailleurs, il pourrait y avoir des spécificités ethniques concernant à fois l'élevage et les habitudes culinaires. Les tests statistiques réalisés sur nos données ne permettent pas de confirmer ces deux premières hypothèses (temps de travail, ethnie). En revanche, les exploitations cotonnières ont à la fois significativement plus d'espèces animales élevées et une alimentation moins diversifiée que les exploitations qui n'ont pas cultivé de coton l'année précédente (test de comparaison de moyennes). De plus, si nous ajoutons dans la régression une variable dichotomique indiquant si l'exploitation a produit du coton en 2012-2013, le nombre d'espèces animales élevées n'est plus significatif. Ainsi, on peut proposer l'interprétation suivante qui s'appuie sur le lien entre le coton et le cheptel : les exploitations cotonnières ont relativement plus de revenus, ce qui leur permet d'acheter plus d'animaux, mais ces revenus ne sont pas principalement dédiés à l'alimentation. Les femmes de ces exploitations consomment en moyenne 0,5 groupes d'aliments de moins que les femmes des exploitations ne produisant pas de coton.

La présence de différentes espèces d'arbres locaux conservés sur les parcelles a un effet très significatif et important sur la diversité alimentaire en mai et août 2013. Ces arbres constituent une source alimentaire importante par leurs fleurs, leurs fruits et leurs graines ou encore les insectes qu'ils abritent (en particulier les chenilles prédatrices du karité). La collecte et la préparation de ces produits alimentaires de cueillette concernent plus les femmes, car elles sont chargées de l'alimentation. Le nombre d'espèces d'arbres n'est pas associé à la diversité alimentaire en janvier 2014, car la période de cueillette est saisonnière : les feuilles et fruits du baobab, les fruits et graines du néré, les feuilles et fleurs du kapokier, les fruits du « raisin sauvage », les feuilles et fleurs de tamarinier, les feuilles et fruits du prunier ou les fruits du rônier se cueillent entre mars et août (notre enquête qualitative et Bergeret et Ribot, 1990). Powell et al. (2011) observent en Tanzanie que $62 \%$ des aliments issus de la nature sont obtenus sur les parcelles des exploitations. Ici, on a observé, en mai 2013, que les exploitations possédant au moins un arbre " sauvage » sont en moyenne $66 \%$ à avoir consommé des fruits ou des légumes feuilles, contre $51 \%(p<0,05)$ pour celles qui n'ont pas d'arbres. Plusieurs études ont déjà montré l'importance des plantes sauvages dans l'alimentation et l'apport en micronutriments (Blaney et al., 2009 au Gabon ; Powell et al., 2013 en Tanzanie). Boedecker et al. (2014) au Bénin observent que les femmes qui consomment des plantes sauvages ont une meilleure diversité alimentaire que celles qui n'en consomment pas. Ils soulignent que les plantes sauvages sont consommées en complément d'autres aliments plutôt qu'en substitution. Ainsi, d'un point de vue nutritionnel, les produits de cueillette cuisinés par les femmes sont importants pour la qualité de l'alimentation.
Par ailleurs, la valeur des ventes, par le chef d'exploitation, des produits agricoles de la saison 2012-2013 est faiblement liée à la diversité de la consommation alimentaire aux trois périodes; ce lien augmente au fur et à mesure que l'on s'éloigne de la date des récoltes. Ces résultats nous interpellent sur deux points : pourquoi l'effet des recettes monétaires agricoles est-il aussi faible, alors que la diversité dépend beaucoup d'achats ( $c f$. paragraphe précédent) ? Et pourquoi cet effet augmente-t-il quand on s'éloigne des récoltes ?

L'effet différencié des recettes agricoles entre les périodes peut s'interpréter par un effet épargne. En mai, il y a encore de l'argent disponible dans la plupart des exploitations et ce facteur n'est pas vraiment discriminant, alors que dans les périodes suivantes, seules les exploitations qui ont gagné suffisamment d'argent en ont encore, car elles ont pu épargner. Concernant l'effet de seuil, la variation des revenus monétaires (agricoles ici) a un lien avec la diversité alimentaire seulement à partir d'un certain niveau d'argent disponible. Si l'on fait la régression avec les quartiles de revenu des ventes agricoles, seul le plus haut quartile est lié à la diversité alimentaire des femmes. Des études plus poussées seraient nécessaires pour valider ces hypothèses interprétatives.

Sur le premier point, le revenu monétaire des ventes des produits agricoles provient essentiellement du coton, et comme nous l'avons expliqué plus haut, le revenu du coton est souvent géré par le chef d'exploitation et n'est pas forcément dédié à l'alimentation.

Par ailleurs, il faut noter que la régression (Tab. 3) inclut les transferts intra-ménages, et que ceux-ci sont corrélés avec le niveau des ventes de produits agricoles. Dans cette région du Burkina Faso, les femmes travaillent dans les champs familiaux qui sont gérés par les chefs d'exploitation (choix des cultures, main-d'œuvre, commercialisation). En retour, ceux-ci fournissent aux femmes des céréales pour la préparation du tô et parfois un peu d'argent, que les femmes dédient surtout à l'alimentation. Les femmes qui ont reçu de tels transferts sont dans des exploitations dont le chef a gagné en moyenne $84000 \mathrm{FCFA}$, soit le double de celles où les femmes n'ont pas reçu de transferts $(p=0,0002)$. Sans la variable Transferts, le coefficient de régression de la variable Ventes des produits agricoles devient faiblement significatif en première période, mais ne change pas fondamentalement de valeur $(0,003 ; p<0,1)$. Pour les autres périodes, l'ordre de grandeur et la significativité restent semblables. Ajouter les transferts dans la régression nous permet d'observer par quel chemin le revenu agricole impacte la diversité alimentaire. Il confirme le fait que, à recette monétaire agricole équivalente, les ménages où les femmes reçoivent un transfert ont plus de chance d'avoir une alimentation diversifiée.

Ici, les recettes agricoles des femmes ne sont liées à la diversité alimentaire qu'en mai 2013, quelques mois après les récoltes, peut-être parce qu'elles sont très faibles (20 000 FCFA en moyenne pour la campagne 2012-2013) et qu'après quelques mois, elles sont entièrement dépensées (contrairement aux revenus du coton, plus importants). En revanche, les revenus non agricoles des femmes et les transferts monétaires de la part des chefs d'exploitation sont en lien très étroit avec la diversité, et ce dernier résultat est valable quelle que soit la période considérée ou la méthode d'analyse utilisée. 
Cela signifie qu'il existe des marges de manœuvre dans la gestion interne des ménages pour améliorer l'alimentation. Ces résultats convergent avec plusieurs autres études concernant les liens entre genre et alimentation au sein des ménages. Hoddinott et Haddad (1995) puis Duflo et Udry (2004) en Côte d'Ivoire, et Doss (2005) au Ghana ont montré que le pouvoir de décision des femmes et leur contrôle sur les ressources (empowerment) sont reliés à l'allocation pour l'alimentation d'une plus grande part des revenus ; Malapit et al. (2015) au Népal ont établi que les femmes ont un meilleur score de diversité alimentaire et des enfants en meilleur état nutritionnel quand elles ont plus de revenus et d'autonomie dans la décision, et/ou quand elles vivent dans des ménages où les pouvoirs entre hommes et femmes sont plus équilibrés.

\section{Conclusion}

Les liens entre la production agricole et la diversité de la consommation alimentaire, indicateur de la qualité nutritionnelle de l'alimentation, passent théoriquement par l'autoconsommation et/ou par les revenus des exploitations agricoles.

Dans le cas des Hauts-Bassins au Burkina-Faso, nous avons montré que produire des cultures plus diversifiées n'était pas corrélé avec une meilleure diversité alimentaire des femmes, probablement parce que les systèmes de production sont peu diversifiés et que les ménages autoconsomment peu, mis à part les céréales. Avoir un revenu monétaire agricole plus élevé la saison précédente est faiblement relié à la diversité de l'alimentation des femmes; ce lien est conditionné à la redistribution à l'intérieur des ménages.

Au-delà des variables agricoles de l'exploitation, qui ont finalement peu d'effets sur la diversité alimentaire, nous avons mis en évidence l'importance de la capacité des femmes à contrôler et à disposer de ressources, à travers des activités agricoles ou non agricoles, des activités de cueillette ou des transferts au sein des ménages.

Cette étude a permis de mettre en évidence certains facteurs agricoles et socioéconomiques associés à la diversité de l'alimentation au sein des ménages agricoles. Leur prise en compte peut permettre d'orienter des interventions agricoles qui seraient plus « sensibles à la nutrition » (Ruel et al., 2013). Les arbres locaux cultivés dans les parcelles agricoles ont un rôle prépondérant dans la diversité alimentaire et les pratiques agricoles qui permettent leur maintien devraient être encouragées. L'autoconsommation des produits agricoles, en dehors des céréales, est limitée, et ni les marchés tels qu'ils sont organisés et fonctionnent actuellement, ni les niveaux de revenus des ménages agricoles ne permettent, en moyenne, d'atteindre un niveau de diversité alimentaire satisfaisant. En revanche, une meilleure répartition des revenus entre hommes et femmes, grâce à des transferts monétaires, au sein des familles, ainsi qu'une plus grande autonomie via des activités génératrices des revenus permettent de compenser - partiellement mais très significativement - ces déficits structurels. Ainsi, des politiques agricoles en faveur d'un plus grand partage des revenus et en faveur de l'autonomie des femmes auraient rapidement un effet sur la diversité alimentaire et la nutrition.

\section{Références}

Arsenault JE, Nikiema L, Alleman P, Ayassou KA, Lanou H, Moursi M, et al. 2014. Seasonal differences in food and nutrient intakes among young children and their mothers in rural Burkina Faso. J Nutr Sci 3: e55.

Bégin F, Frongillo EA, Delisle H. 1999. Caregiver behaviors and resources influence child height-for-age in rural Chad. J Nutr 129 (3): 680-686.

Bergeret A, Ribot J. 1990. L'arbre nourricier en pays sahélien. Paris: Éditions de la Maison des sciences de l'homme.

Bhagowalia P, Headey D, Kadiyala S. 2012. Agriculture, income, and nutrition linkages in India: insights from a nationally representative survey. In: IFPRI Discussion Paper 01195.

Blaney S, Beaudry M, Latham M. 2009. Contribution of natural resources to nutritional status in a protected area of Gabon. Food Nutr Bull 30(1): 49-62.

Boedecker J, Termote C, Assogbadjo A, Van Damme P, Lachat C. 2014. Dietary contribution of wild edible plants to women's diets in the buffer zone around the Lama forest, Benin - an underutilized potential. Food Secur 6(6): 833-849. doi: 10.1007/ s12571-0140396-7.

Bricas N, Akindes F. 2012. Afrique de l'Ouest. In: Poulain J.P. (ed.). Dictionnaire des cultures alimentaires. Paris: PUF, p. 21-30.

Carletto G, Ruel M, Winters P, Zezza A. 2015. Farm-level pathways to improved nutritional status: introduction to the special issue. $J$ Dev Stud 51: 651-957.

Cilss. 2004. Vingt ans de prévention des crises alimentaires au Sahel. Bilan et perspectives. Ouagadougou: Cilss.

Dillon A, McGee K, Oseni G. 2014. Agricultural production, dietary diversity, and climate variability. In: World Bank, Policy Research Working Paper No. 7022.

Doss C. 2005. The effects of intrahousehold property ownership on expenditure patterns in Ghana. J Afr Econ 15(1): 149-180. doi: 10.1093/jae/eji025.

Duflo E, Udry C. 2004. Intrahousehold resource allocation in Cote d'Ivoire: social norms, separate accounts and consumption choices. In: National Bureau of Economic Research Working Paper No. 10498

Dury S, Bocoum I. 2012. Le paradoxe de Sikasso : pourquoi produire plus ne suffit-il pas pour bien nourrir les enfants des familles d'agriculteurs. Cah Agric 21(5): 324-336.

Hoddinott J, Haddad L. 1995. Does female income share influence household expenditures? Evidence from Côte d'Ivoire. Oxford: Oxford Bulletin of Economics and Statistics, pp. 77-95.

Jones D, Shrinivas A, Bezner-Kerr R. 2014. Farm production diversity is associated with greater household dietary diversity in Malawi: findings from nationally representative data. Food Policy 46: $1-12$.

Kelly VA, Tefft JF, Oehmke JF, Staatz JM. 2004. Identifying policy relevant variables for reducing childhood malnutrition in rural Mali. In: Department of Agricultural Economics Staff Paper Series, Michigan State University. Available from http://purl.umn.edu/11528.

Lourme-Ruiz A, Maugérard E. 2014. Le paradoxe des Hauts-Bassins : produire plus pour nourrir mieux ? Film documentaire 42 minutes, version française et anglaise, disponible sur https://vimeo.com/ 120670833.

Malapit HJ, Kadiyala S, Quisumbing A, Cunningham K, Tyagi P. 2015. Women's empowerment mitigates the negative effects of low production diversity on maternal and child nutrition in Nepal. J Dev Stud 51: 1097-1123

Martin-Prével Y, Allemand P, Wiesmann D, Arimond M, Ballard T, Deitchler M, et al. 2015. Moving forward on choosing a standard 
operational indicator of women's dietary diversity. Rome: Food and Agriculture Organization of the United Nations.

Miller D, Welch R. 2013. Food system strategies for preventing micronutrient malnutrition. Food Policy 42: 83-93.

Parent G, Zagré NM, Ouédraogo A, Guiguembé TR. 2002. Les grands hydro-aménagements au Burkina Faso contribuent-ils à l'amélioration des situations nutritionnelles des enfants ? Cah Agric 11(1): $51-57$.

Powell B, Hall J, Johns T. 2011. Forest cover, use and dietary intake in the East Usambara Mountains, Tanzania. Int For Rev 13(3): 305-324.

Powell B, Maundu P, Kuhnlein HV, Johns T. 2013. Wild foods from farm and forest in the East Usambara Mountains, Tanzania. Ecol Food Nutr 52(6): 451-478. doi: 10.1080/03670244.2013.768122.

Ruel MT, Alderman H, and the Maternal and Child Nutrition Study Group. 2013. Nutrition-sensitive interventions and programmes: how can they help to accelerate progress in improving maternal and child nutrition? Lancet 382(9891): 536-551. doi: 10.1016/S01406736(13)60843-0.

Sibhatu T, Krishna V, Qaim M. 2015. Production diversity and dietary diversity in smallholder farm households. Proc Natl Acad Sci USA 112(34): 10657-10662.

StataCorp. 2011. BT Stata statistical software: release 12. College Station, TX: StataCorp LP.

Tefft J, Penders C, Kelly V, Staatz JM, Yade M, Wise V. 2000. Linkages between agricultural growth and improved child nutrition in Mali. In: Michigan State University International Development Working Papers, 79 p. xiii-50.

Torheim LE, Ouattara F, Diarra MM, Thiam FD, Barikmo I, Hatløy A, et al. 2004. Nutrient adequacy and dietary diversity in rural Mali: association and determinants. Eur J Clin Nutr 58: 594-604. doi: $10.1038 /$ sj.ejen.1601853.

Citation de l'article : Lourme-Ruiz A, Dury S, Martin-Prével Y. 2016. Consomme-t-on ce que l'on sème ? Relations entre diversité de la production, revenu agricole et diversité alimentaire au Burkina Faso. Cah. Agric. 25: 65001. 